\title{
Weg von napoleonischen und wilhelminischen Modellen! Hin zu einer demokratischen Justizverfassung, auch in Deutschland!
}

Das Resümee von Horst Häuser lautet: Die deutsche Justiz ist in einer vorkonstitutionellen, vordemokratischen Weise verfasst. Für diese autoritär und hierarchisch strukturierte Form der Justizorganisation und -verwaltung hat der italienische Kollege Salvatore Senese 1982 den Begriff »napoleonisches Modell« verwendet. Ich selbst habe mich und die interessierte Öffentlichkeit 1997 gefragt: »Mit Kaisers Justiz ins dritte Jahrtausend? « Ich stimme Horst Häuser zu: So ist es. Und ich kann und werde - in meinem ersten Teil (I.) - dem Elend noch ein i-Tüpfelchen hinzufügen (1.). Dann allerdings werde ich - auch insoweit durchaus mit ihm übereinstimmend, auch wenn er mir diesen Part überlassen hat -, sechs Elemente des Rechts und der Wirklichkeit der deutschen Justiz benennen, die so etwas wie demokratie-adäquate Inselchen in dem napoleonischen oder wilhelminischen Meer darstellen (2.) und die, wie ich dann im zweiten Teil (II.) zeigen werde, im Sinne eines demokratie-adäquaten Gesamtsystems ausbaufähig sind.

\section{Generelles Elend und wenige Glanzpunkte im status quo}

\section{Die Verordnung des Reichsjustizministers von 1935 als Krücke der heutigen Gerichtsorganisation}

Wenn es um den Behördenaufbau in der Justiz geht (in der Ordentlichen Gerichtsbarkeit in den Ländern etwa das Verhältnis von Amtsgericht, Landgericht und Oberlandesgericht), stößt der suchende Jurist, der nach Grundgesetz und Rechtsprechung des Bundesverfassungsgerichts gewohnt ist, eine »verfassungsfeste« gesetzliche Grundlage zu suchen, auf nichts oder allenfalls auf einen Trümmerhaufen.

Sie können, jedenfalls für die Ordentliche Gerichtsbarkeit und vorbehaltlich genauerer Länderregelungen, nichts finden außer der Verordnung zur einheitlichen Regelung der Gerichtsverfassung (GVVO) des Reichsjustizministers (!) vom 20. März 1935 (RGBl. I, S. 403), die an sich nur rudimentär und übergangsweise die organisatorischen Verhältnisse der Gerichte und die dienstrechtlichen Verhältnisse der Richter und Staatsanwälte regeln sollte, nachdem die Zuständigkeit für die Justiz von den Ländern auf das Reich übergegangen war. Dort und nur dort werden Sie etwas finden zur Frage der Dienstaufsicht über die Richter (Art. VIII, § 16) und die Dienstaufsicht über die Behörden ( $(14)$, über die Zuständigkeit in Dienstaufsichtsbeschwerdeangelegenheiten ( $§ 17)$; sie finden dort auch Details, etwa, dass dem Landgerichtspräsidenten die Dienstaufsicht über ein mit einem Präsidenten besetztes Amtsgericht nicht zusteht (§ 14 Abs. 2). Der Präsident des Amtsgerichts verkehrt dann also mit der Präsidentin des OLG (Ich spreche von Schleswig-Holstein) direkt und nicht über den Präsidenten des Landgerichts. Das ist z. B. in Fragen der Personalverteilung sehr relevant. 
Nun ist diese Verordnung allerdings mit Wirkung vom 24.04.2008 in Zusammenhang mit der vom Kollegen Richter am Bundesverwaltungsgericht Bernd Brunn im Bundesministerium der Justiz (BMJ) vorbereiteten großen Rechtsbereinigung (Gesetz vom 19.04.2006, BGBI I S. 866) als Bundesrecht aufgehoben worden. Man nimmt an, dass sie, soweit die Zuständigkeit der Länder als Träger der Landesgerichte berührt ist, als Landesrecht fortgilt.

Aber wie bereits angemerkt: Es ist nicht zu fassen, ausgerechnet die inneren Angelegenheiten der Justiz und die innerdienstlichen Verhältnisse der Richter (derjenigen also, die penibel auf die Einhaltung der »Wesentlichkeitstheorie« achten, die an Gesetz und Recht (Art. 20 Abs. 3 GG) gebunden sind) sind fast überhaupt nicht oder, wenn überhaupt, durch eine Verordnung aus der Hand des Reichsjustizministers Gürtner von 1935 geregelt.

Ein Grund mehr, an die Reform zu gehen!

Ein ironischer Nachtrag: Wenn man den nicht nur anachronistischen, sondern auch noch, rechtsstaatlich gesehen, rudimentären Charakter des Rechts der Gerichtsorganisation beklagt, so muss man direkt die klare gesetzliche Regelung für die Staatsanwaltschaft loben. Freilich ist diese nun, jedenfalls dem geschriebenen Recht nach, vollends wilhelminisch. Ich spreche von den $\S 141$ bis 147 (für die Bundesanwälte zusätzlich $\S \S 148$ bis 151) des Gerichtsverfassungsgesetzes (GVG).

\section{Die Lichtblicke in der Düsternis}

Ich habe sechs Punkte ausgemacht, die man als demokratie-adäquate Ausgangspunkte für eine zukünftige weitestgehend selbstverwaltete Justiz ansehen könnte.

(1) Die Präsidien der Gerichte (für unsere europäischen Gäste: eine Art geschäftsführender Ausschuss des Plenums der Richter) haben bereits jetzt die wichtigste Leitungsaufgabe eines Gerichts zu erfüllen, nämlich die Zusammensetzung der Spruchkörper und die richterliche Geschäftsverteilung. Die Präsidien haben seit 2000 überdies ein verbessertes Wahlrecht. Es ist nunmehr auch geklärt, dass sie richteröffentlich tagen dürfen.

(2) Als Vorstufe richterlicher Selbstverwaltung gibt es, freilich in den Ländern unterschiedlich ausgestaltet nach Intensität und Zuständigkeitsfeldern, Formen der Mitbestimmung (wiederum für unsere ausländischen Freunde: Richterräte wie in den Unternehmen die Betriebsräte). Die richterliche Mitbestimmung wird im Einzelnen durch die Richter- und Präsidialräte ausgeübt. Vereinfacht gesagt: Die Richterräte sind im Allgemeinen für die Mitbestimmung zuständig, die Präsidialräte bei sogenannten Beförderungen.

(3) a) Für vorbildlich sehe ich auch schon jetzt die Garantie der persönlichen Freiheiten der Richter, auch als Staatsbürger, an ( $\$ 39$ des Deutschen Richtergesetzes (DRiG), fälschlich häufig als Mäßigungsgebot bezeichnet). Richtigerweise kann man ihm entnehmen, dass der Richter/die Richterin grundsätzlich die vollen staatsbürgerlichen Rechte behält (juge citoyen). Grenze ist nur die Kollision mit einer Beeinträchtigung der richterlichen Unabhängigkeit. Hier ist richterliche Eigenverantwortlichkeit gefragt. In Grenz- und Streitfällen muss - durch Gerichte - über Besorgnis der Befan- 
genheit entschieden werden. Oder, worauf ich gleich kommen werde, es müssen die Richterdienstgerichte als Disziplinarinstanz entscheiden.

b) Was die Disziplinarangelegenheiten angeht, sind für diese nur auf der untersten Stufe (Verweis) die Präsidenten als Dienstvorgesetzte zuständig, ansonsten entscheiden hierüber mit Richtern besetzte, wiederum vom Präsidium eines Gerichts bestimmte Richter. Überdies hat die einzelne Richterin/der einzelne Richter die Möglichkeit, nach $\S 26$ Abs. 3 DRiG von sich aus das Richterdienstgericht anzurufen, um, wie bereits angesprochen, eine streitige Frage der Grenzen und des Umfanges der Dienstaufsicht einerseits und der richterlichen Freiheiten andererseits klären zu lassen.

(4) Ein Schutz der richterlichen Unabhängigkeit, wenn auch nicht (jedenfalls nicht in erster Linie) in der Form der Selbstverwaltung, liegt in dem besonderen Verfahren, nach dem in Deutschland im Bund (dort verbindlich) und in zahlreichen Ländern (diesen ist es nach dem Grundgesetz freigestellt) die Richterinnen und Richter in ihr Amt berufen werden. Dies erfolgt nämlich nicht durch den zuständigen Minister (in der Regel: den Justizminister) allein, sondern im Zusammenwirken mit parlamentarischen Richterwahlausschüssen, in denen zum Teil auch Richter mitwirken, also: Stellvertretend für das Volk wählt das Parlament die Richter und gibt Ihnen so einen höheren Grad der demokratischen Legitimation und gewährleistet zugleich stärker die Pluralität; in anderen Ländern der Bundesrepublik sind es nach wie vor allein die Justizminister. In jedem Falle aber nehmen - siehe oben unter »Mitbestimmung « -, die Präsidialräte als Organ der Mitbestimmung vor Auswahl und Ernennung Stellung.

(5) Ein weiterer Punkt, der nach dem deutschen Grundgesetz die Unabhängigkeit der Richterinnen und Richter schützt - dies wird Sie an dieser Stelle vielleicht überraschen -, ist Art. 100 Abs. 1 GG. Er lautet: »Hält ein Gericht ein Gesetz, auf dessen Gültigkeit es bei der Entscheidung ankommt, für verfassungswidrig, so ist das Verfahren auszusetzen und ... die Entscheidung des Bundesverfassungsgerichtes einzuholen.« Der Richter legt dann die Sache unmittelbar dem Bundesverfassungsgericht vor. Ein »Dienstweg « besteht hier nicht. Ein Dazwischentreten insbesondere der Exekutive ist also ausgeschlossen. Auch dies stärkt, bei der Anwendung und Auslegung des Rechts nach den Maßstäben der deutschen Verfassung (und daneben durchaus auch der EMRK), die Unabhängigkeit des einzelnen Richters.

(6) Als Letztes sei noch die besondere Richterbesoldung erwähnt. Mag sie auch noch immer hierarchische Elemente in sich bergen - mein Kollege Carsten Löbbert wird nachher näher darüber sprechen - so ist sie doch ansatzweise schon auf eine Annäherung und - im Idealfall - Vereinheitlichung der Richtergehälter angelegt. Wir können das vielleicht in der Diskussion nach dem Referat Löbbert vertiefen. Jedenfalls ist die Richterbesoldung gegenüber der Beamtenbesoldung differenziert und trägt den Besonderheiten des Richteramtes Rechnung.

Nota bene: Es existiert bereits heute in Deutschland das Muster eines selbstverwalteten und auch in Verwaltungsangelegenheiten von der Exekutive vollständig unabhängigen Gerichts: das Bundesverfassungsgericht. Das Plenum dieses Gerichts entscheidet alle Angelegenheiten selbst oder durch Kommissionen. Der Präsident ist Geschäftsführer des Plenums. Das Gericht ist nicht dem Bundesjustizministerium 
nachgeordnet. Es verkehrt unmittelbar mit den übrigen Verfassungsorganen, insbesondere dem Finanzminister und dem Parlament.

Es sei der Vollständigkeit halber darauf hingewiesen, dass diese Statusfragen nicht von vornherein im Sinne einer vollständigen - auch verwaltungsmäßigen - »Selbstregierung « des Gerichts geregelt waren, vielmehr haben sich die Richterinnen und Richter des Gerichts diese im »Statusstreit« in den fünfziger Jahren erkämpft.

\section{Eine grundlegende Reform der Justiz in ihrem Inneren und im Verhältnis zur Regierung und Parlament steht auch in Deutschland auf der Tagesordnung.}

Eine grundlegende Reform muss aus folgenden Elementen bestehen (wie ich sie im Folgenden in Anlehnung an das Konzept der Richterinnen und Richter, Staatsanwältinnen und Staatsanwälte in ver.di vortrage).

1. Die Kompetenzen der Gerichtspräsidien als Selbstverwaltungsorgane sind in Richtung einer Zuständigkeit für alle personellen und organisatorischen Angelegenheiten des Gerichts und seiner Angehörigen auszubauen.

Die Selbstverwaltung ist »von unten nach oben« auszubauen, d. h. auf der Ebene der größeren Gerichtsbezirke (in der ordentlichen Gerichtsbarkeit: LG, OLG) und auf Landesebene sind Selbstverwaltungsorgane zu schaffen (zur Landesebene s. unter 4.).

2. Präsidentinnen und Präsidenten (oder Direktorinnen und Direktoren) sind - wie die Inhaber aller Funktionsstellen - zukünftig auf Zeit zu wählen. Sie führen insgesamt die Geschäfte des Präsidiums.

Das »Beförderungswesen« wird abgeschafft. Alle Richterämter sind gleichwertig und werden grundsätzlich gleich besoldet.

Funktionsstellen sind auf Zeit zu besetzen. Die Lebenszeitwahl und -ernennung für das Richteramt als solches sollte unberührt bleiben.

Das formelle Vorsitzendenamt in Spruchkörpern entfällt.

3. Die rechtlichen und tatsächlichen Kompetenzen der parlamentarischen Richterwahlausschüsse sind im Bund und - soweit vorhanden - in den Ländern zu stärken. Wo noch nicht vorhanden, sind parlamentarische Richterwahlausschüsse einzuführen. Persönlich neige ich dazu, »reine« Richterwahlausschüsse zu bevorzugen (also solche, die nur aus Parlamentariern bestehen). In Einklang mit dem Vorschlag meiner Organisation, den Richterinnen und Richter in der Vereinten Dienstleistungsgewerkschaft (ver.di) sage ich aber auch hier: Dem Richterwahlausschuss sollen auch Richterinnen und Richter angehören, die - auf Vorschlag der Richterschaft - ebenfalls vom Parlament gewählt werden.

Auch wenn die Vorschläge von ver.di und NRV einen Parlamentarischen Richterwahlausschuss (jedoch mit richterlicher Komponente) bevorzugen und das Modell des DRB Justizwahlausschüsse vorsieht (mit insgesamt sehr knapper nichtrichterlicher Mehrheit, nämlich nur wegen der Stimme des Vorsitzenden, der nicht Richter ist), so lassen sich doch die Modelle kompatibel machen: Es besteht Einigkeit, dass jedenfalls nicht das (reine) System der Selbstergänzung (Kooptation) bestehen soll, wenn es eines Tages die Selbstverwaltung der Justiz auch in Deutschland gibt. 
Um noch einmal meine persönliche Meinung deutlich zu sagen: Die historische Errungenschaft »Richterwahlausschuss « sollte auf keinen Fall aufgegeben werden. Im Gegenteil: Richterwahlausschüsse müssen endlich in den Ländern, in denen es sie noch nicht gibt, eingeführt werden. Ein zukünftiger Art. 98 Abs. 4 GG muss dies für die Länder für verbindlich erklären.

4. a) Für Angelegenheiten der Justizverwaltung auf Landesebene und für die Bundesgerichte sind Gerichtsbarkeitsräte als oberste Verwaltungsorgane der Justiz einzurichten. Sie übernehmen die Vorbereitung des Haushalts und die Kommunikation mit dem Finanzministerium und dem Parlament, also Aufgaben, die bisher den Justizministerien obliegen.

Interessant wird es sein, die Frage zu klären, wie die Landgerichtsbarkeitsräte und der Gerichtsbarkeitsrat für die Bundesgerichte insgesamt auf Bundesebene koordiniert werden. Hier liegt ein Problem, das sich speziell im föderalen Staat stellt. Soweit mir bekannt, sind die Modelle in anderen Ländern im Wesentlichen auf zentrale Strukturen zugeschnitten. Hier wird man etwa Modelle der Aufsichts- und Leitungsgremien unserer öffentlich-rechtlichen Rundfunk- und Fernsehanstalten (ARD und ZDF), Strukturen der Programmkommission der Deutschen Richterakademie oder - auf dem Gebiet der Hochschul- und Bildungsverwaltung - die Modelle »Rektorenkonferenz«, »Ständige Konferenz der Kultusminister« (KMK), Deutscher Akademischer Austauschdienst (daad) oder Pädagogischer Austauschdienst (PAD) auf ihre Ausbaufähigkeit und Übertragbarkeit überprüfen müssen.

b) Wichtig in diesem Zusammenhang: Eine ausreichende Finanzausstattung der Justiz insgesamt ist sicherzustellen. In anderen Staaten werden hierzu bestimmte Messzahlen, ausgehend vom Bruttosozialprodukt, für eine Mindestausstattung verwendet.

c) Die (verbleibenden) Aufgaben der Justizministerien werden neu definiert. Hierzu werden etwa gehören:

- Justiziariat der Regierung,

- Gesetzgebungsangelegenheiten,

- Koordinierung der Gesetzgebung und verbleibender Justizministerialangelegenheiten im Verhältnis Bund/Länder,

- Justizvollzug,

- Gnadenwesen und

- Notardienstaufsicht, um nur einige zu nennen. Dies wird im Detail weiter zu klären sein.

5. Für die Gerichtsbarkeitsräte sind zum einen, auf Vorschlag der Richterschaft, Richterinnen und Richter vom Parlament zu wählen; sie bilden die Mehrheit. Daneben sind, ebenfalls vom Parlament, Parlamentarier oder andere von ihm gewählte Vertreter oder Vertreterinnen der gesellschaftlichen Pluralität und/oder der juristischen Fachprofessionen (z. B. Anwälte, Professoren) als Mitglieder vorzusehen.

Die Vorschläge aus der Richterschaft für die vom Parlament zu wählenden richterlichen Mitglieder werden nach dem Verhältniswahlrecht getroffen. Jede Richterin/jeder Richter hat gleiches Stimmrecht. Gleiches gilt für die Richterinnen und Richter, die von der Richterschaft als Mitglieder der Richterwahlausschüsse vorgeschlagen werden (s. oben unter 3.). 
Nota bene auch hier (aber zu einem ganz anderen, sehr wesentlichen Punkt):

Ich habe hier nur über die Gerichte gesprochen. Dies konnte ich umso freier, da in der rechtspolitischen Diskussion auf europäischer wie auf deutscher Ebene Modelle für die Staatsanwaltschaft vorliegen und hier auf dieser Tagung auch vorgetragen werden. Auch ich halte es für an der Zeit, die, wie ich schon sagte, durch und durch wilhelminische normative Ordnung unserer Staatsanwaltschaft dem demokratischen Geist des Grundgesetzes anzupassen. Und zum Glück kann ich ja hier anfügen: der schon weitgehend viel liberaleren Praxis anzugleichen. Aber entscheidend geht es darum, gesichertes Recht zu schaffen, das der Bindung der Staatsanwältinnen und Staatsanwälte wie der Richterinnen und Richter an Verfassung, Gesetz und Recht gerecht wird und ihnen gerade deshalb und dafür das, wie ich meine, selbstverständliche Recht gibt, die eigenen Angelegenheiten beider Zweige der »Dritten Gewalt« demokratisch organisiert selbst zu verwalten.

\section{Wie soll das alles ins Werk gesetzt werden?}

Wenn - hoffentlich bald - die politische Umsetzungsreife für die verstärkte Selbstverwaltung der Justiz erreicht ist, sollte man bundesrechtlich eine Regelung vorsehen, die es den Ländern (und dem Bund für dessen Gerichte) ermöglicht, mit gutem Beispiel voranzugehen (»Experimentierklausel"). So könnte ein Wettbewerb entstehen, an dessen Ende ein ausgereiftes Gesamtmodell stünde.

\section{Zum Weiterlesen:}

Hans-Ernst Böttcher, »Mit Kaisers Justiz ins dritte Jahrtausend? «, in: SchleswigHolsteinische Anzeigen 1997, S. $101 \mathrm{ff}$.

Ders., Vorbemerkungen vor Art. 92 ff »Justiz in der Bundesrepublik DeutschlandRichterbild, Justizstruktur, Erforderlichkeit von Reformen «, in GG, Mitarbeiterkommentar und Handbuch, hrsg. von Dieter C. Umbach und Thomas Clemens, Heidelberg (C. F. Müller) 2002

Ders., »Reform der Justizstrukturen - technokratisch-fiskalisch oder substantiell? «, in: Schleswig-Holsteinische Anzeigen 2004, S. 253 ff. (ursprünglich in: Neue Kriminalpolitik 2004, S. $28 \mathrm{ff.}$.)

Ders., Autogoverno e cogestione del tribunali nella Repubblica federale tedesca (Selbstverwaltung und Mitbestimmung der Gerichte in der Bundesrepublik Deutschland), in: Pier Luigi Zanchetta (Hrsg.), Governo e autogoverno della magistratura nell' Europa occidentale (Verwaltung und Selbstverwaltung der Justiz in Westeuropa), Milano (Franco Angeli) 1987

Sicherung der Unabhängigkeit der Richterinnen und Richter durch verstärkte Selbstverwaltung der Gerichte und der Justiz (Position der Richterinnen und Richter, Staatsanwältinnen und Staatsanwälte in ver.di, vom 10.12.2003, in: verdikt 2.03, S. $19 f$. 